\title{
STUDY OF SERUM MAGNESIUM LEVEL IN TYPE 2 DIABETES MELLITUS
}

\author{
Nagarajan $K^{1}$
}

${ }^{1}$ Professor, Department of Medicine, Sri Manakula Vinayagar Medical College and Hospital.

\begin{tabular}{l}
\hline ABSTRACT \\
\hline BACKGROUND \\
A high prevalence of magnesium deficiency is reported in diabetics. Magnesium depletion has a negative impact on glucose \\
homoeostasis and insulin sensitivity in type 2 diabetic patients as well as on the evolution of complications such as retinopathy, \\
nephropathy, neuropathy and arterial atherosclerosis. \\
The aim of this study is to estimate the prevalence of hypomagnesaemia in patients with type 2 diabetes mellitus and its \\
correlations with microvascular complications of diabetes like retinopathy, nephropathy, neuropathy.
\end{tabular}

\section{MATERIALS AND METHODS}

Patients with type 2 diabetes admitted in Thanjavur Medical College and Hospital over a period of one year from October 2011 to October 2012 formed the study population. The sample size was 100 patients. Serum magnesium concentration was measured by calmagite dye method.

\section{RESULTS}

The study revealed that prevalence of hypomagnesaemia in study subjects was 35\%. Sex, age and duration of diabetes were not significant predictors of serum magnesium. Significant association was found between hypomagnesaemia and diabetic retinopathy, nephropathy, neuropathy. Significant correlations were not found with comorbidities such as ischaemic heart disease and hypertension. Low serum magnesium concentrations are common in type 2 diabetics. Magnesium deficiency is conclusively associated with diabetic retinopathy, nephropathy, neuropathy.

\section{KEYWORDS}

Magnesium, Diabetes Mellitus, Retinopathy, Nephropathy, Neuropathy.

HOW TO CITE THIS ARTICLE: Nagarajan K. Study of serum magnesium level in type 2 diabetes mellitus. J. Evolution Med. Dent. Sci. 2017;6(32):2619-2626, DOI: 10.14260/Jemds/2017/566

BACKGROUND
India is frequently referred to as the diabetic capital of the world as it has the highest number of cases in the world.

Worldwide, in the last 2 decades, incidence has suddenly increased from 30 million cases in 1985 to 171 million in 2000. Recent data suggests that prevalence of DM by the year 2030 could be 360 million. DM is worldwide in distribution and the incidence of both types is rising. ${ }^{1,2}$

The distribution of both T1DM and T2DM varies worldwide, due to relative difference in genetic and environmental factors in different parts of the world. Recent data shows it is associated with $10-30 \%$ reduction of life expectancy, most common cause of blindness in the age group of 20 to 65 years, 25-fold increased risk of nontraumatic lower limb amputations and increased incidence of end stage renal disease approximately 1000 patients per year. ${ }^{2}$ India had around 31.7 million cases in year 2000 which is expected to rise alarmingly to around 79.4 million in 2030 by which time every fifth diabetic subject in the world would be an Indian. ${ }^{3}$

In Tamilnadu, the prevalence in 2008 is $18.6 \%$ in urban areas and $9.1 \%$ in rural areas. ${ }^{3}$

Financial or Other, Competing Interest: None.

Submission 16-04-2016, Peer Review 05-04-2017,

Acceptance 13-04-2017, Published 20-04-2017.

Corresponding Author:

Dr. Nagarajan K,

No. 5, Professor Quarters,

Sri Manakula Vinayagar Medical College and Hospital,

Madagadipet, Puducherry.

E-mail: tnjknagarajan@gmail.com

DOI: $10.14260 /$ jemds $/ 2017 / 566$
Although DM can cause hypomagnesaemia, low serum magnesium level is a risk factor for DM. Magnesium is an essential element for several enzymes that plays important role in glucose metabolism. Animal studies found that low magnesium has a negative effect on post receptor signalling of insulin. Some studies have also found that magnesium supplementation improves insulin action and glucose metabolism in diabetics. ${ }^{4}$

Magnesium is involved in multiple levels of insulin secretion. Magnesium deficiency can modify the $\mathrm{Na}+\mathrm{K}+$ ATPase channel that maintains sodium, potassium and glucose transport. 5

In DM, there is a direct correlation between serum magnesium concentration in blood and cellular glucose disposal that is independent of insulin secretion. ${ }^{6}$

Low serum magnesium level has direct correlation with microvascular complications (Retinopathy, ${ }^{7}$ neuropathy. nephropathy) and macrovascular complications (IHD and cerebrovascular disease). ${ }^{8}$

In elderly type $2 \mathrm{DM}$, Paolisso hypothesised that oral magnesium supplementation for 4 weeks results in decreased fasting blood sugar level, increased plasma and $\mathrm{RBC}$ magnesium level. ${ }^{9}$

In this study, serum magnesium concentration in type 2 DM patients is correlated with occurrence of microvascular complications.

\section{Objectives}

\section{The Aims of this study are}

1. Measurement of serum magnesium concentration in diabetes mellitus. 
2. To estimate the prevalence of hypomagnesaemia in patients with type 2 diabetes mellitus.

3. Its correlation with microvascular complications like retinopathy, nephropathy, neuropathy.

\section{MATERIALS AND METHODS \\ Source of Data}

Patients with type 2 diabetes admitted in Thanjavur Medical College \& Hospital, Thanjavur who satisfied the inclusion criteria and consented to participate in the study were included.

\section{Period of Study}

October 2015-October 2015.

\section{Type of Study}

\section{Descriptive Study}

100 patients were randomly selected of which 50 were male and 50 were female. Cases with renal failure, acute coronary syndromes, patients on diuretics, alcoholics or with malabsorption were excluded. None were taking magnesium supplements or magnesium containing antacids. Informed consent was obtained.

\section{Inclusion Criteria}

All patients both males and females above 13 years of age group with type 2 diabetes mellitus admitted in Internal Medicine units of Thanjavur Medical College \& Hospital, Thanjavur.

\section{Exclusion Criteria}

1. Patients with chronic renal failure.

2. Acute myocardial infarction in last 6 months.

3. Patients on diuretics.

4. Patients with history of alcohol abuse.

5. Patients receiving magnesium supplements or magnesium containing antacids.

6. Malabsorption or chronic diarrhoea.

7. Age $<13$ years.

\section{Data Collection}

The 100 diabetics (50 Men \& 50 Women) were included in the study. Detailed history - including duration of diabetes, treatment mode, symptoms suggestive of diabetic neuropathy, associated diseases such as hypertension and ischaemic heart disease was obtained as per the proforma.

Detailed physical and neurological examination was done. Retinopathy was assessed by direct ophthalmoscopy.

Samples were collected for estimation of fasting blood glucose and magnesium level. Postprandial blood sugar was measured two hours after a standard meal. Nerve conduction study was done on selected patients by an experienced neurologist with symptoms \& signs suggestive of neuropathy. Blood urea, serum creatinine and 24-hour urinary albumin were estimated. Serum magnesium was estimated by calmagite dye method. HbA1C measurement done by a modified calorimetric method.

\section{Calmagite Dye Method- Test Principle}

Under alkaline conditions, magnesium combines with calmagite dye to form a red colour which is read spectrophotometrically at $530 \mathrm{~nm}$. Formation of colour depends on magnesium levels. To eliminate the interference of calcium during estimation, EDTA is included in the reagent. Cyanide reduces heavy metal interference. Surfactant reduces protein interference.

\section{Test Procedure}

Three test tubes labelled Blank, Standard and Test are prepared as in table.

\begin{tabular}{|c|c|c|c|}
\hline In test tubes & Blank & Standard & Test \\
\hline Calmagite & $1 \mathrm{~mL}$ & $1 \mathrm{~mL}$ & $1 \mathrm{~mL}$ \\
\hline Standard sample & - & $10 \mathrm{~mL}$ & - \\
\hline Patient's sample & - & - & $10 \mathrm{~mL}$ \\
\hline Distilled water & $10 \mathrm{~mL}$ & - & - \\
\hline
\end{tabular}

This test tubes are incubated at room temperature (22$28^{\circ} \mathrm{C}$ ). The absorbance of Test (A) T, Standard (A) S and Blank (A) B are read at $530 \mathrm{~nm}$ in TSB spectrophotometer. Magnesium concentration is calculated by the following formula.

Magnesium concentration $\left.(\mathrm{mEq} / \mathrm{L})=\underset{\mathrm{T}}{\left(\mathrm{A}_{\mathrm{B}}-\mathrm{A}_{\mathrm{S}} / \mathrm{A}_{\mathrm{B}}\right.}-\mathrm{A}\right)$

Serum magnesium concentration is expressed in $\mathrm{mg} / \mathrm{dL}$ by linearity of $1 \mathrm{mEq} / \mathrm{L}=1.2 \mathrm{mg} / \mathrm{dL}$.

According to Magnesium Levels Patients were classified into-

1. Normal 1.7 to $2.4 \mathrm{mg} / \mathrm{dL}$,

2. Low $<1.7 \mathrm{mg} / \mathrm{dL}$,

3. High $>2.4 \mathrm{mg} / \mathrm{dL}$.

Patients were also categorised on the basis of duration of diabetes, presence of ischaemic heart disease or hypertension, mode of treatment, presence/absence of retinopathy, neuropathy and nephropathy, and glycaemic control (FBS and HbA1C).

\section{Cases with Diabetic Retinopathy were Further Divided into}

a. Non-proliferative diabetic retinopathy.

b. Proliferative diabetic retinopathy.

Diabetic Nephropathy was graded depending on 24-hour Urinary Excretion of Albumin as follows-

No nephropathy, $<30 \mathrm{mg} / 24$ hours.

Microalbuminuria $30-299 \mathrm{mg} / 24$ hours.

Macroalbuminuria (clinical proteinuria) $>=300 \mathrm{mg} / 24$ hours

\section{Statistical Analysis}

The statistical analysis was done by SPSS 15 software. MS Word and Excel were used to generate tables and charts.

Following tests were used-

- Chi square test.

- Student T test.

- One way ANOVA test.

Statistical results were considered significant at $\mathrm{P}<0.05$. 


\section{RESULTS}

100 cases of type 2 DM (50 males, 50 females, mean age 56.87 years) comprised the study group.

\begin{tabular}{|c|c|}
\hline Age (in years) & No. of Patients (n = 100) \\
\hline Below 50 yrs. & 30 \\
\hline 51 to 60 yrs. & 41 \\
\hline 61 to 70 yrs. & 22 \\
\hline 71 yrs. \& above & 7 \\
\hline \multicolumn{2}{|c|}{ Table 1. Age Distribution } \\
\hline
\end{tabular}

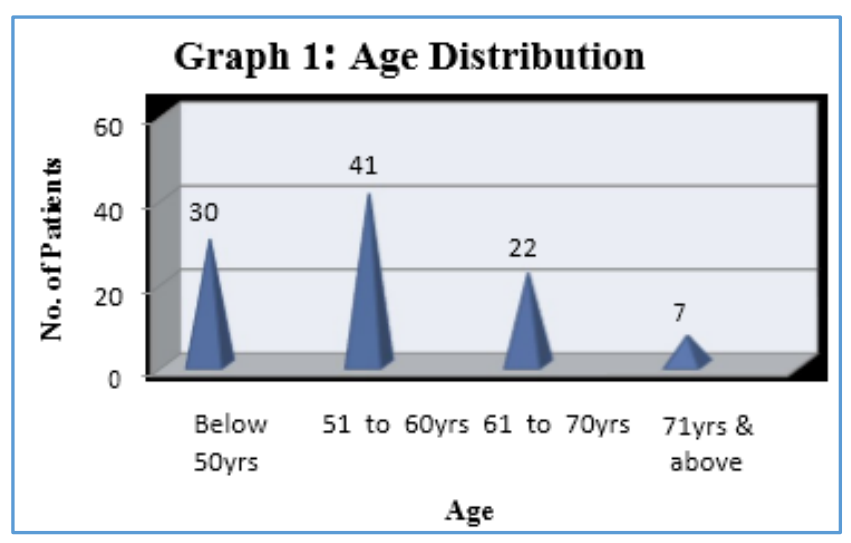

Patients were distributed across the age spectrum of 42 to 78 years. Mean age 56.87 years. Most patients $(n=41)$ were present in 51-60 years age group. Youngest patient was 42 years old.

\begin{tabular}{|c|c|}
\hline \multicolumn{2}{|c|}{ Characteristics } \\
\hline No. of Subjects & 100 \\
\hline Age (Years) & $56.87(42-78)$ \\
\hline Men & 50 \\
\hline Women & 50 \\
\hline Duration of diabetes & \multirow{2}{*}{$8.89(2-23)$} \\
\hline (Years) & \\
\hline \multicolumn{2}{|l|}{ Medication } \\
\hline Oral Hypoglycaemics & 67 \\
\hline Insulin and Oral & \multirow{2}{*}{33} \\
\hline Hypoglycaemics & \\
\hline Diet only & 0 \\
\hline \multicolumn{2}{|l|}{ Comorbidities } \\
\hline Hypertension & 30 \\
\hline Ischaemic Heart Disease & 15 \\
\hline \multicolumn{2}{|l|}{ Diabetic Retinopathy } \\
\hline NPDR & 33 \\
\hline PDR & 2 \\
\hline Diabetic Neuropathy & 15 \\
\hline \multicolumn{2}{|l|}{ Diabetic Nephropathy } \\
\hline Microalbuminuria & 29 \\
\hline Macroalbuminuria & 4 \\
\hline Poor glycaemic control & 39 \\
\hline \multicolumn{2}{|c|}{ Table 2. Characteristics of Study Population } \\
\hline
\end{tabular}

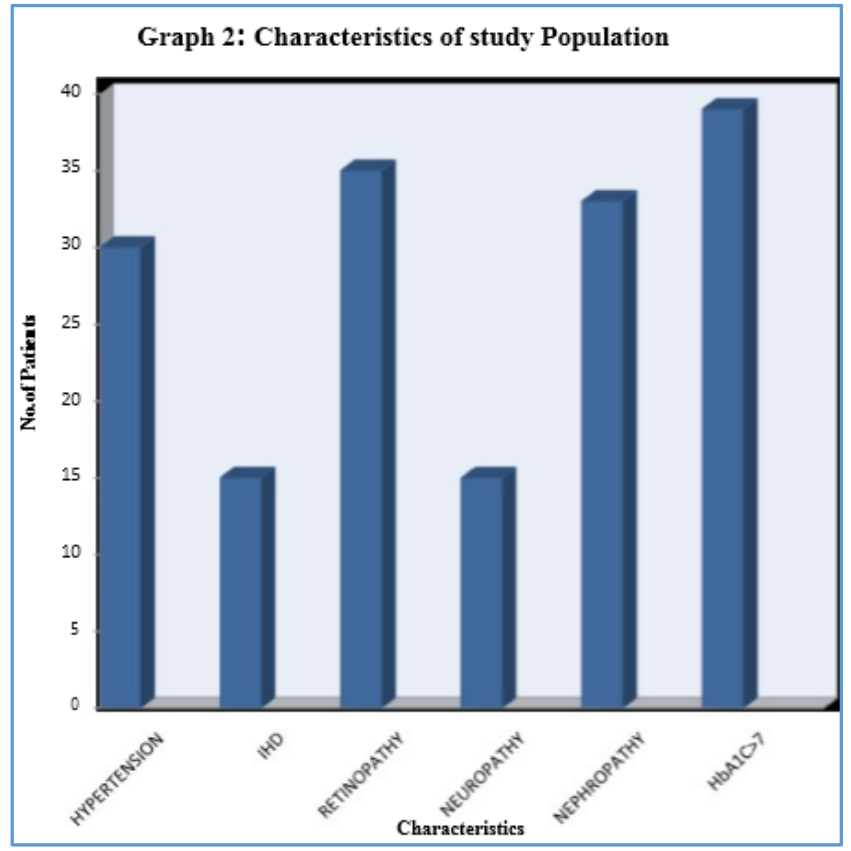

The average duration of diabetes in study population was 8.89 years and range was 2 years to 23 years. 67 patients received only oral hypoglycaemic agents and 33 patients received both. 30 patients had hypertension and 15 patients had ischaemic heart disease and 55 patients had no comorbidities. Total 35 patients had diabetic retinopathy. Total of 15 patients had diabetic neuropathy. 33 patients had nephropathy.

\begin{tabular}{|c|c|c|c|}
\hline & \multicolumn{2}{|c|}{ Magnesium } & \\
\hline Sex & $\begin{array}{c}\text { Normomagnesaemia } \\
(n=65)\end{array}$ & $\begin{array}{c}\text { Hypomagnesaemia } \\
(n=35)\end{array}$ & $\begin{array}{l}\text { Statistical } \\
\text { Inference }\end{array}$ \\
\hline \multirow{3}{*}{ Male } & \multirow{3}{*}{$34(52.3 \%)$} & \multirow{3}{*}{$16(45.7 \%)$} & $\mathrm{X}^{2}=.396$ \\
\hline & & & $\mathrm{Df}=1$ \\
\hline & & & $.529>0.05$ \\
\hline & & & $\begin{array}{c}\text { Not } \\
\text { Significant }\end{array}$ \\
\hline Female & $31(47.7 \%)$ & $19(54.3 \%)$ & \\
\hline
\end{tabular}

\begin{tabular}{|c|c|c|c|c|}
\hline $\begin{array}{r}\text { Sl. } \\
\text { No }\end{array}$ & MG & Mean & S.D & $\begin{array}{c}\text { Statistical } \\
\text { Inference }\end{array}$ \\
\hline 1 & Male (n = 50) & 0.492 & 0.7253 & T=-.622 df=98 \\
\cline { 3 - 5 } & & $.536>0.05$ \\
\hline 2 & Female (n = 50) & 0.584 & 0.7547 & Not Significant \\
\hline \multicolumn{4}{|c|}{ Table 3. Prevalence of Hypomagnesaemia } \\
\hline
\end{tabular}

\section{Graph 3: Prevalence of Hypomagnesemia}

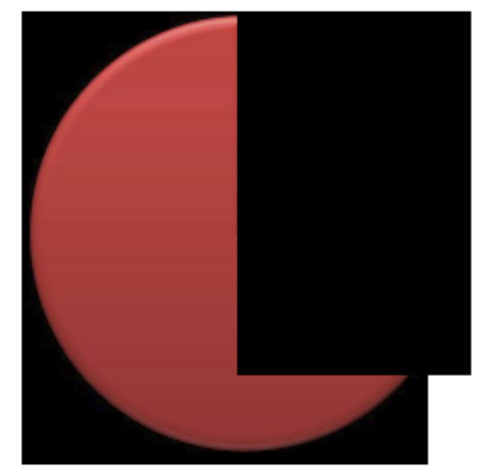

Hypomagnesemia 35\%

Normomagnesemia $65 \%$ 
Hypomagnesaemia was found in 35 patients. 65 patients had normomagnesaemia No patient had hypermagnesaemia. No correlation was found between hypomagnesaemia in men and women ( $45.7 \%$ and $54.3 \% \%$ respectively).

\begin{tabular}{|c|c|c|c|c|c|c|c|}
\hline Sl. No & MG & Mean & S.D & SS & Df & MS & Statistical Inference \\
\hline 1 & Between Groups & & & 32.917 & 4 & 8.229 & \multirow{7}{*}{$\begin{array}{c}\mathrm{F}=13.265 \\
0.060(>0.05) \\
\text { Not } \\
\text { Significant }\end{array}$} \\
\hline 2 & Below 5 yrs. $(n=45)$ & 0 & 0 & & & & \\
\hline 3 & 6 to 10 yrs. $(n=25)$ & 0.804 & 0.7898 & & & & \\
\hline 4 & 11 to 15 yrs. $(n=15)$ & 1.4333 & 0.4012 & & & & \\
\hline 5 & 16 to 20 yrs. $(n=10)$ & 1.22 & 0.646 & & & & \\
\hline 6 & 21 yrs. $\&$ above $(n=5)$ & 0 & 0 & & & & \\
\hline 7 & Within Groups & & & 20.979 & 95 & 0.221 & \\
\hline
\end{tabular}

\begin{tabular}{|c|c|c|c|c|}
\hline \multirow{2}{*}{ Sl. No. } & \multirow{2}{*}{$\begin{array}{c}\text { Duration } \\
\text { (Years) }\end{array}$} & $\begin{array}{c}\text { Mormomagnesaemia } \\
\text { (n= 65) }\end{array}$ & $\begin{array}{c}\text { Hypomagnesaemia } \\
\text { (n= 35) }\end{array}$ & $\begin{array}{c}\text { Statistical } \\
\text { Inference }\end{array}$ \\
\cline { 2 - 4 } & Below 5 yrs. & $46(70.8 \%)$ & $2(5.7 \%)$ & $\mathrm{X}^{2}=4.568$ \\
$\mathrm{Df}=4$ & $0.067(>0.05)$ \\
\hline 1 & 6 to 10 yrs. & $12(18.5 \%)$ & $13(37.1 \%)$ & Not \\
\hline 3 & 11 to 15 yrs. & $1(1.5 \%)$ & $8(22.9 \%)$ & Significant \\
\hline 4 & 16 to 20 yrs. & $2(3.1 \%)$ & $1(2.9 \%)$ & \\
\hline 5 & 21 yrs. \& above & $4(6.2 \%)$ & & \\
\hline
\end{tabular}

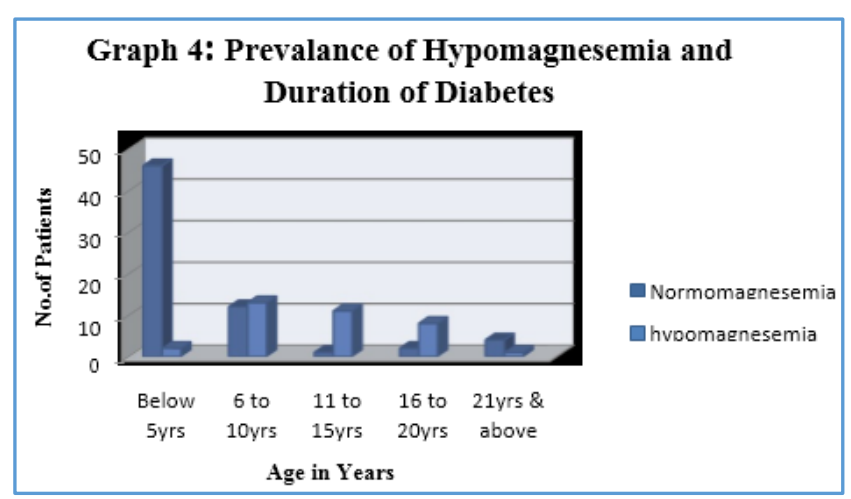

Chi- square $(\chi 2)$ value is 4.568 . So, the correlation is insignificant $p$ value. So the duration of diabetes does not significantly predict serum magnesium concentration. One way ANOVA $\mathrm{F}=13.265$ in between groups also shows no significant correlation. The mean duration was 8.89 years (2-23).

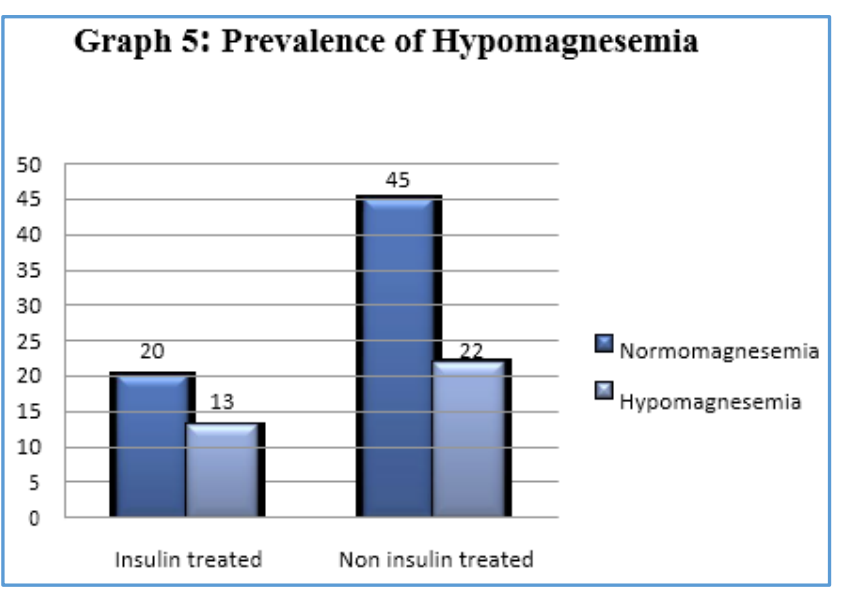

Insulin treated - Non-insulin treated

\begin{tabular}{|c|c|c|c|c|}
\hline \multirow[b]{2}{*}{$\begin{array}{l}\text { Sl. } \\
\text { No }\end{array}$} & \multirow[b]{2}{*}{ FBS } & \multicolumn{2}{|c|}{ Magnesium } & \multirow[b]{2}{*}{$\begin{array}{l}\text { Statistical } \\
\text { Inference }\end{array}$} \\
\hline & & $\begin{array}{l}\text { Normomagne- } \\
\text { saemia } \\
(n=65)\end{array}$ & $\begin{array}{l}\text { Hypomagne- } \\
\text { saemia } \\
(n=35)\end{array}$ & \\
\hline 1 & Below 90 & $8(12.3 \%)$ & $2(5.7 \%)$ & \multirow{6}{*}{$\begin{array}{c}\mathrm{X}^{2}=45.582 \\
\mathrm{Df}=5 \\
0.000 \\
(<0.05) \\
\text { Significant }\end{array}$} \\
\hline 2 & 91 to 100 & $11(16.9 \%)$ & 0 & \\
\hline 3 & 101 to 110 & $10(15.4 \%)$ & $9(25.7 \%)$ & \\
\hline 4 & 111 to 120 & $19(29.2 \%)$ & $1(2.9 \%)$ & \\
\hline 5 & 121 to 130 & $13(20 \%)$ & $2(5.7 \%)$ & \\
\hline 6 & $\begin{array}{l}131 \& \\
\text { above }\end{array}$ & $4(6.2 \%)$ & $21(60 \%)$ & \\
\hline & & Fasting Bl & gar & and \\
\hline
\end{tabular}

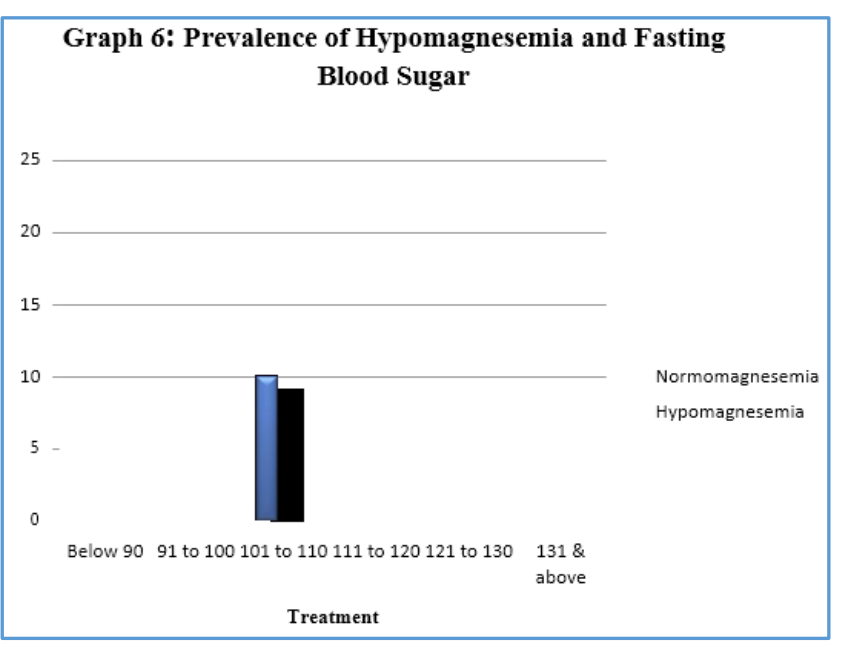

Prevalence of hypomagnesaemia is high when fasting blood sugar $>131(60 \%)$. The chi-square $(\chi 2)$ value is 45.582 . So the correlation is significant. So, fasting blood sugar can significantly predict serum magnesium concentration. 


\begin{tabular}{|c|c|c|c|c|}
\hline \multirow[b]{2}{*}{$\begin{array}{l}\text { Sl. } \\
\text { No. }\end{array}$} & \multirow[b]{2}{*}{ HbA1C } & \multicolumn{2}{|c|}{ Magnesium } & \multirow[b]{2}{*}{$\begin{array}{l}\text { Statistical } \\
\text { Inference }\end{array}$} \\
\hline & & $\begin{array}{l}\text { Normomagne- } \\
\text { saemia } \\
(n=65)\end{array}$ & $\begin{array}{c}\text { Hypomagne- } \\
\text { saemia } \\
(n=35)\end{array}$ & \\
\hline 1 & Below 6 & $8(12.3 \%)$ & $2(5.7 \%)$ & \multirow{6}{*}{$\begin{array}{c}\mathrm{X}^{2}=10.408 \\
\mathrm{Df}=5 \\
0.064(>0.05) \\
\text { Not } \\
\text { Significant }\end{array}$} \\
\hline 2 & 6 to 7 & $18(27.7 \%)$ & $5(14.3 \%)$ & \\
\hline 3 & 7 to 8 & $15(23.1 \%)$ & $5(14.3 \%)$ & \\
\hline 4 & 8 to 9 & $5(7.7 \%)$ & $9(25.7 \%)$ & \\
\hline 5 & 9 to 10 & $8(12.3 \%)$ & $4(11.4 \%)$ & \\
\hline 6 & $10 \&$ above & $11(16.9 \%)$ & $10(28.6 \%)$ & \\
\hline
\end{tabular}

Serum magnesium concentration showed no significant association with $\mathrm{HbA1C}$. Chi square $\mathrm{X}^{2}=10.408, \mathrm{Df}=5, \mathrm{P}$ value 0.064 . Higher prevalence of hypomagnesaemia observed in HbA1c > 10 (28.6\%).

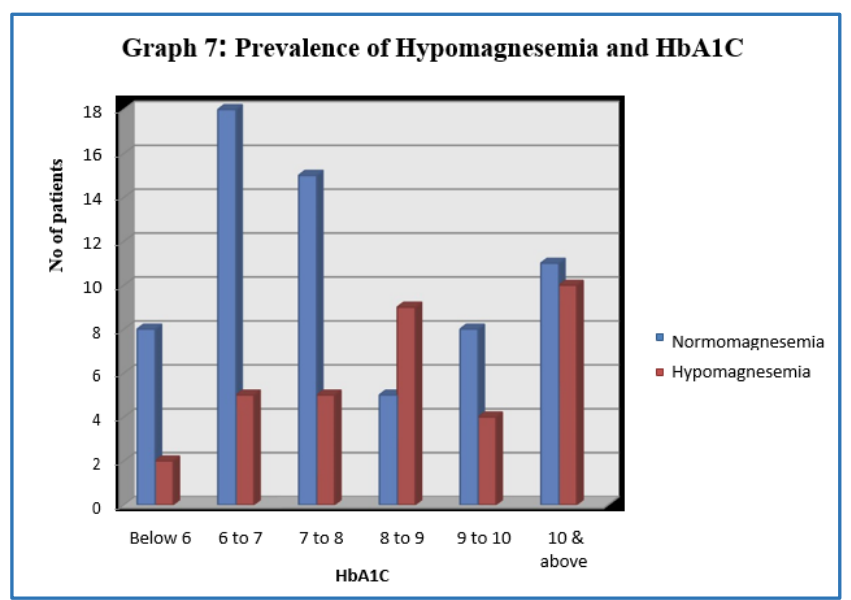

Graph 7. Prevalence of Hypomagnesaemia and HbA1C

\begin{tabular}{|c|c|c|}
\hline & No. of Patients & Percentage \\
\hline Particulars & $(\mathrm{n}=100)$ & $100 \%$ \\
\hline No Retinopathy & 65 & 65 \\
\hline NPDR & 33 & 33 \\
\hline PDR & 2 & 2 \\
\hline \multicolumn{2}{|c|}{ Table 8. Prevalence of Hypomagnesaemia } \\
and Diabetic Retinopathy \\
\hline
\end{tabular}

\begin{tabular}{|c|c|c|c|c|}
\hline \multirow[b]{2}{*}{$\begin{array}{l}\text { Sl. } \\
\text { No. }\end{array}$} & \multirow[b]{2}{*}{$\begin{array}{c}\text { Retino- } \\
\text { pathy }\end{array}$} & \multicolumn{2}{|c|}{ Magnesium } & \multirow[b]{2}{*}{$\begin{array}{l}\text { Statistical } \\
\text { inference }\end{array}$} \\
\hline & & $\begin{array}{c}\text { Normomagne- } \\
\text { saemia } \\
(n=18)\end{array}$ & $\begin{array}{c}\text { Hypomagne } \\
\text { saemia } \\
(n=17)\end{array}$ & \\
\hline 1 & NPDR & $18(100 \%)$ & 15 (88.2\%) & $\begin{array}{c}X^{2}=4.746 \\
D f=1\end{array}$ \\
\hline 2 & PDR & 0 & $2(11.8 \%)$ & $\begin{array}{c}0.034(<0.05) \\
\text { Significant }\end{array}$ \\
\hline
\end{tabular}

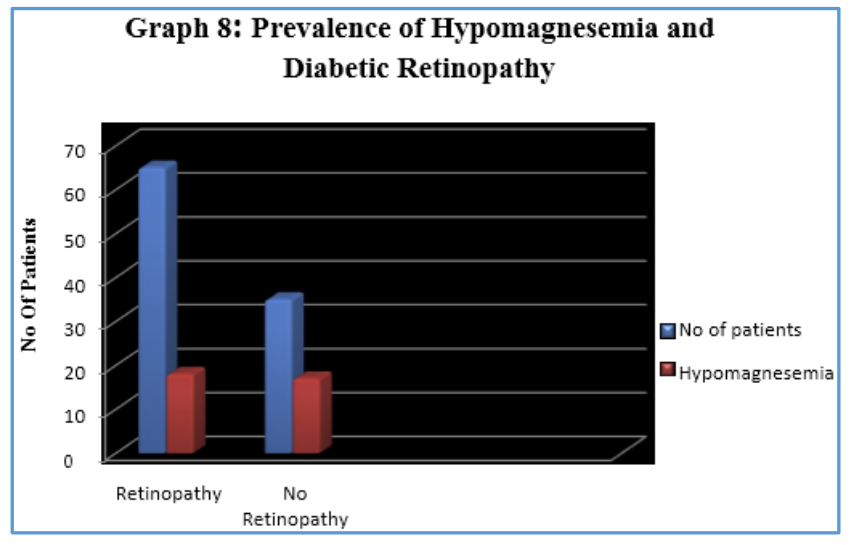

Observations revealed a definite correlation between hypomagnesaemia and diabetic retinopathy. The chi-square $(\chi 2)$ value is $4.746 . \mathrm{Df}=1 . \mathrm{p}$ value is 0.034 .

\begin{tabular}{|c|c|c|}
\hline Particulars & $\begin{array}{c}\text { No. of patients } \\
(\mathbf{n = 1 0 0 )}\end{array}$ & $\begin{array}{c}\text { Percentage } \\
(\mathbf{1 0 0 \% )}\end{array}$ \\
\hline No neuropathy & 85 & 85 \\
\hline neuropathy & 15 & 15 \\
\hline
\end{tabular}

\begin{tabular}{|c|c|c|c|c|}
\hline \multirow{2}{*}{$\begin{array}{c}\text { Sl. } \\
\text { No. }\end{array}$} & Neuropathy & $\begin{array}{c}\text { Normomagne- } \\
\text { saemia } \\
(\mathbf{n = 6 5 )}\end{array}$ & $\begin{array}{c}\text { Hypomagne- } \\
\text { saemia } \\
\text { (n=35) }\end{array}$ & $\begin{array}{l}\text { Statistical } \\
\text { Inference }\end{array}$ \\
\hline 1 & Negative & $60(92.3 \%)$ & $25(71.4 \%)$ & $\begin{array}{c}\mathrm{X}^{2}=7.778 \\
\mathrm{Df}=1 \\
\mathrm{n}=1\end{array}$ \\
\hline 2 & Positive & $5(7.7 \%)$ & $10(28.6 \%)$ & $\begin{array}{c}0.005(<0.05) \\
\text { Significant }\end{array}$ \\
\hline \multicolumn{3}{|r|}{ Table 9. Prevalence of Hypomagnesaemia } \\
and Diabetic Neuropathy
\end{tabular}

Observations revealed a definite correlation between hypomagnesaemia and diabetic neuropathy. The chi-square $(\chi 2)$ value is $7.778, \mathrm{Df}=1, \mathrm{p}$ value is 0.005 .

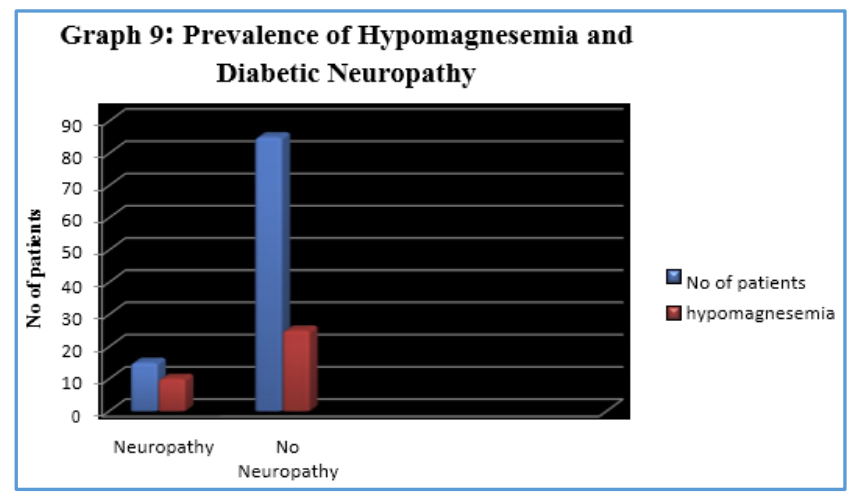

\begin{tabular}{|c|c|c|}
\hline & No. of Persons & Percentage \\
\hline Particulars & $(\mathrm{n}=100)$ & $(100 \%)$ \\
\hline No albuminuria & 67 & 67 \\
\hline Macroalbuminuria & 4 & 4 \\
\hline Microalbuminuria & 29 & 29 \\
\hline \multicolumn{2}{|c|}{$\begin{array}{c}\text { Table 10. Prevalence of Hypomagnesaemia } \\
\text { and Diabetic Nephropathy }\end{array}$} \\
\hline \multicolumn{2}{|c|}{} \\
\hline
\end{tabular}




\begin{tabular}{|c|c|c|c|c|}
\hline \multirow[b]{2}{*}{$\begin{array}{l}\text { Sl. } \\
\text { No. }\end{array}$} & \multirow[b]{2}{*}{ Nephropathy } & \multicolumn{2}{|c|}{ Magnesium } & \multirow[b]{2}{*}{$\begin{array}{l}\text { Statistical } \\
\text { Inference }\end{array}$} \\
\hline & & $\begin{array}{c}\text { Normoma- } \\
\text { gnesaemia } \\
(n=65)\end{array}$ & $\begin{array}{c}\text { Hypomagne- } \\
\text { saemia } \\
(n=35)\end{array}$ & \\
\hline \multirow{3}{*}{1} & \multirow{3}{*}{ No albuminuria } & \multirow{3}{*}{65 (100\%) } & \multirow{3}{*}{$2(5.7 \%)$} & $\mathrm{X}^{2}=91.471$ \\
\hline & & & & $\mathrm{Df}=2$ \\
\hline & & & & $0.000(<0.05)$ \\
\hline 2 & Macroalbuminuria & 0 & $4(11.4 \%)$ & \\
\hline 3 & Microalbuminuria & 0 & $29(82.9 \%)$ & Significant \\
\hline
\end{tabular}

\begin{tabular}{|c|c|c|c|c|}
\hline \multirow{2}{*}{$\begin{array}{c}\text { Sl. } \\
\text { No. }\end{array}$} & SHT & $\begin{array}{c}\text { Normo- } \\
\text { magnesaemia } \\
(\mathbf{n}=\mathbf{6 5})\end{array}$ & $\begin{array}{c}\text { Hypomagne- } \\
\text { saemia } \\
\text { (n = 35) }\end{array}$ & $\begin{array}{c}\text { Statistical } \\
\text { Inference }\end{array}$ \\
\hline \multirow{2}{*}{1} & Absent & $43(66.2 \%)$ & $27(77.1 \%)$ & $\mathrm{X}^{2}=1.308$ \\
\cline { 3 - 5 } & & & $\mathrm{Df}=1$ \\
\hline 2 & Present & $22(33.8 \%)$ & $8(22.9 \%)$ & Not Significant \\
\hline \multicolumn{3}{|c|}{ Table 12. Prevalence of Hypomagnesaemia and } \\
Hypertension
\end{tabular}

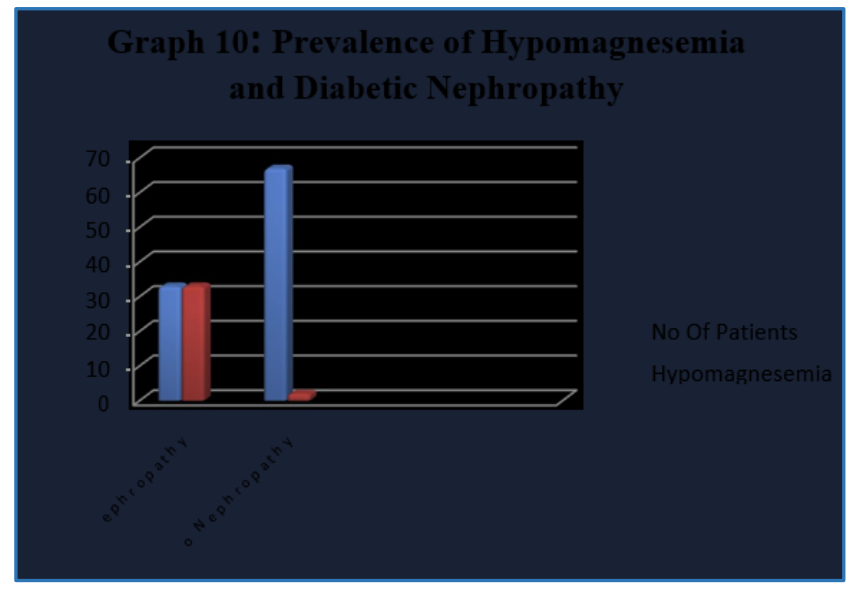

Serum magnesium concentration showed no significance with systemic hypertension, $\mathrm{X}^{2}=1.308, \mathrm{Df}=1$. $\mathrm{P}$ value is insignificant.

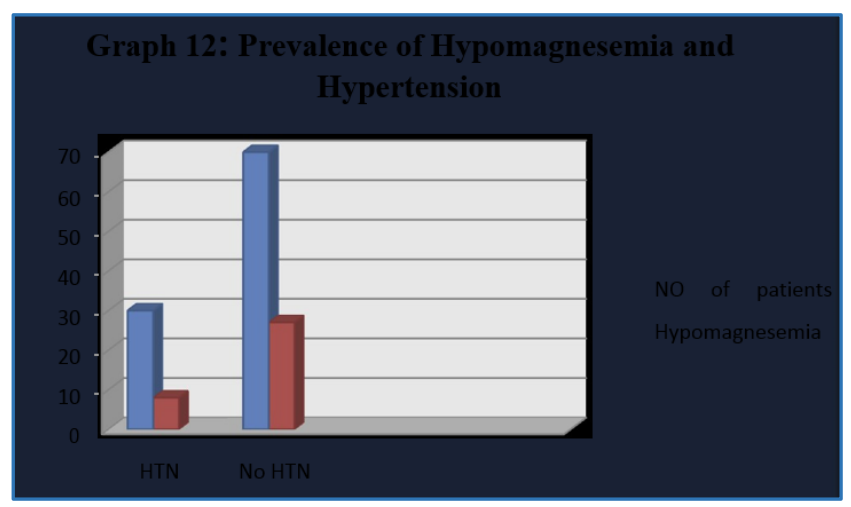

Observations revealed a definite correlation between hypomagnesaemia and diabetic nephropathy. The chi-square $(\chi 2)$ value is 91.471. $\mathrm{Df}=2$.

\begin{tabular}{|c|c|c|c|c|}
\hline \multirow[b]{2}{*}{$\begin{array}{l}\text { Sl. } \\
\text { No. }\end{array}$} & \multirow[b]{2}{*}{ IHD } & \multicolumn{2}{|c|}{ Magnesium } & \multirow[b]{2}{*}{$\begin{array}{l}\text { Statistical } \\
\text { Inference }\end{array}$} \\
\hline & & $\begin{array}{c}\text { Normomagne- } \\
\text { saemia } \\
(n=65)\end{array}$ & $\begin{array}{c}\text { Hypomagne- } \\
\text { saemia } \\
(n=35)\end{array}$ & \\
\hline 1 & Absent & $54(83.1 \%)$ & $31(88.6 \%)$ & \multirow{2}{*}{$\begin{array}{c}\mathrm{X} 2=.539 \\
\mathrm{Df}=1 \\
0.463(>0.05) \\
\text { Not Significant }\end{array}$} \\
\hline 2 & Present & 11 (16.9\%) & $4(11.4 \%)$ & \\
\hline & Tabl & $\begin{array}{l}\text { Prevalen } \\
\text { nd Ischaen }\end{array}$ & $\begin{array}{l}\text { ypomag } \\
\text { art Diseo }\end{array}$ & mia \\
\hline
\end{tabular}

Serum magnesium concentration showed no significant relation with ischaemic heart disease, $\mathrm{X}^{2}=.539, \mathrm{Df}=1$. $\mathrm{P}$ value is insignificant.

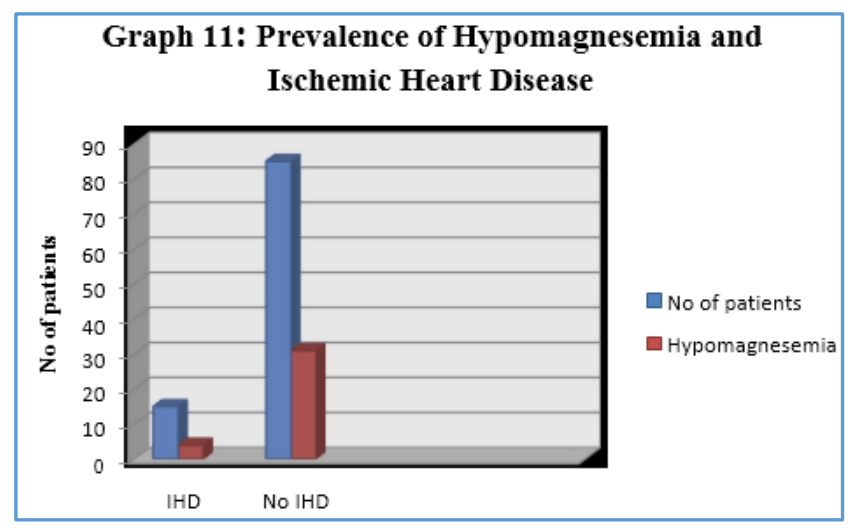

\begin{tabular}{|c|c|c|}
\hline & $\begin{array}{c}\text { Hypo- } \\
\text { magnesaemia }\end{array}$ & $\begin{array}{c}\text { Normo- } \\
\text { magnesaemia }\end{array}$ \\
\hline Complications & $(\mathrm{No}=35)$ & $(\mathrm{No}=65)$ \\
\hline IHD & $4(11.4 \%)$ & $11(16.9 \%)$ \\
\hline HTN & $8(22.9 \%)$ & $22(33.8 \%)$ \\
\hline Retinopathy & $35(48.6 \%)$ & $18(27.7 \%)$ \\
\hline Neuropathy & $10(28.6 \%)$ & $5(7.7 \%)$ \\
\hline Nephropathy & $33(94.3 \%)$ & 0 \\
\hline FBS 131 \& Above & $21(60 \%)$ & $4(6.2 \%)$ \\
\hline \multicolumn{2}{|c|}{ Table 13. Distribution of Complications } \\
\hline
\end{tabular}

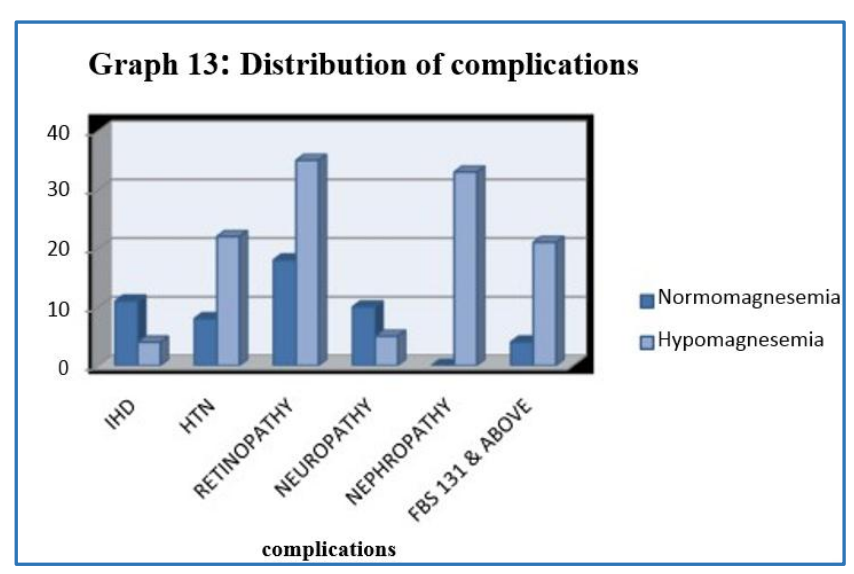

Among the persons with low serum magnesium level prevalence rates of IHD, HTN, Diabetic neuropathy, Diabetic nephropathy, Diabetic Retinopathy and Fasting glucose levels were compared to normomagnesaemic group. Study shows increased incidence of Diabetic neuropathy, Diabetic nephropathy, Diabetic Retinopathy in hypomagnesaemic patients compared to normomagnesaemics. 


\section{DISCUSSION}

Reports of increased prevalence of low plasma magnesium concentrations among diabetic patients and possible association of hypomagnesaemia with diabetic complications prompted this study.

Marked magnesium deficiency has been reported in the previous case series of type-2 DM. However, some workers have also reported normal and even high levels. The present study shows serum magnesium level of 35 cases with type 2 $\mathrm{DM}$, below the reference range. This confirms to the reported prevalence of low plasma magnesium status in T2DM in several studies, which ranged from $13.5 \%$ to $47.7 \%$. Prevalence of hypomagnesaemia in T2 DM in our study was similar to that reported by Nadler et al. ${ }^{10}$ Walti MK et al ${ }^{11}$ also reported a $37.6 \%$ prevalence of hypomagnesaemia in T2DM versus $10.9 \%$ in nondiabetic controls in Zurich, Switzerland.

Serum magnesium concentration though less sensitive is a highly specific indicator of low magnesium status.

Low dietary intake is an unlikely cause of impaired magnesium status in diabetes. A dietary assessment conducted in Europe showed that only 5.4 percentage of the diabetics and 9.1 percentage of the control group had magnesium intake less than reference range. In addition, recently it has been shown that controlled diabetics have absorption of magnesium to a similar as healthy controls. Increased urinary magnesium excretion due to hyperglycaemia and osmotic diuresis may contribute to hypomagnesaemia in diabetes.

Serum levels of magnesium have been found by several investigators to correlate inversely with fasting blood glucose concentration and the percentage of HbA1C. Schlienger et $\mathrm{al}^{12}$ hypothesised that patients with uncontrolled diabetics showed low serum magnesium concentration. The present study also revealed statistically significant correlation between serum magnesium levels and fasting blood sugar (Prevalence of hypomagnesaemia high in fasting blood sugar value above $131 \mathrm{mg} \%$ is $60 \%$ ), but there is no significant correlation with HbA1C. However, a higher prevalence of hypomagnesaemia is observed in $\mathrm{HbA1c}>10$ (28.6\%) in the present study.

Hypomagnesaemia is reported to be both a cause and result of poor glycaemic control. Magnesium is necessary for glucose entry into the cell and act as a cofactor for enzymes of carbohydrate metabolism. In addition, magnesium deficiency has been shown to promote insulin resistance in multiple studies.

Nadler et $\mathrm{al}^{10}$ have reported that insulin sensitivity decreases even in nondiabetic individuals after induction of magnesium deficiency. Likewise, elderly subjects were shown to have improved glucose tolerance when they received magnesium supplements. Thus, hypomagnesaemia by itself results in poor glycaemic control.

Conversely, hyperglycaemia and osmotic diuresis may lead to increased urinary magnesium excretion and hypomagnesaemia in diabetics. However, high prevalence of hypomagnesaemia is reported in type 2 diabetics with good glycaemic control. So although poor glycaemic control is associated with magnesium deficiency, it is not simply induced by hyperglycaemia and is not corrected by improvement in metabolic control alone.

Sex, age duration of diabetes were not the significant predictors of serum magnesium levels. Yajnick et al in 1984 reported that among patients with DM, serum magnesium levels depend on age and males had higher serum magnesium levels than females. The increasing magnesium levels with age were probably due to impaired renal function and the sample size (87 diabetics, 30 nondiabetics) was relatively small to confirm male preponderance. In our study, patients with impaired renal functions were excluded. Our results confirm to the recent reports that have not shown any significant associations between sex, age and duration of diabetes with serum magnesium levels.

Significant differences, in serum magnesium concentrations have been reported between the insulin treated and non-insulin treated diabetics. Our study revealed prevalence of hypomagnesaemia is low in insulin treated patients compared to non-insulin treated patients. ((37.1\% $\mathrm{v} / \mathrm{s} 62.9 \%)$. Yajnik et al reported that insulin treated diabetics have significantly lower serum magnesium levels compared to non-insulin treated ones. However, the difference was statistically not significant. Walti MK et al have reported that diabetes treatment (insulin or OHA) did not significantly predict hypomagnesaemia. Insulin mediates entry of magnesium from plasma to RBC. In a recent study, Alzaida et al $^{13}$ have found that cellular uptake of magnesium is normally stimulated by insulin. So, insulin treatment may enhance cellular magnesium uptake and result in increased prevalence of hypomagnesaemia.

In our study, no association was found between incidence of ischaemic heart disease and hypomagnesaemia. However, several observational studies have reported hypomagnesaemia is associated with higher risk of ischaemic heart disease. As part of atherosclerosis risk in communities study, a cohort of 15,792 subjects were studied over 7 years and an increasing relative risk of coronary artery disease with decreasing serum magnesium was reported. ${ }^{14}$ How a low serum magnesium predisposes to coronary artery disease is not identified yet. However, in our study, no difference in prevalence of hypomagnesaemia was found between those with ischaemic heart disease and others. Similarly, no difference in prevalence of hypomagnesaemia was found between the hypertensive and non-hypertensive subjects.

Trials have shown that magnesium deficiency has been associated with diabetic microvascular disease. In our study, increased prevalence of hypomagnesaemia was observed in diabetics with microvascular complications and mean serum concentration of magnesium in diabetics with microvascular complications was comparatively lower than in diabetics with no microvascular complications.

Hypomagnesaemia has been reported in patients with diabetic retinopathy, with lower magnesium levels predicting a greater risk of severe diabetic retinopathy. Our observations revealed a definite association between diabetic retinopathy and lower serum magnesium levels. There was a significant difference in prevalence of hypomagnesaemia in diabetics with retinopathy and without retinopathy $(48.6 \%$ Vs. 27.7\%; $\mathrm{P}<0.005$ ). These observations are similar to other reports. Grafton et $\mathrm{al}^{15}$ have proposed the inositol transport theory to explain this association. But the exact reason remains obscure.

Hypomagnesaemia is seen in cases with diabetic neuropathy, with lower magnesium levels predicting a greater risk of severe diabetic neuropathy. The present study 
revealed patients with diabetic neuropathy had a slightly higher prevalence of hypomagnesaemia compared to those without neuropathy (28.6\% v/s 7.7\%). Rodriguez- Moran and Guerrero-Romero hypothesised that low serum magnesium level causes high incidence of diabetic foot ulcers.

Hypomagnesaemia is seen in cases with diabetic nephropathy. Decreased magnesium levels predict an increased risk of severe diabetic nephropathy. The present study shows patients with diabetic nephropathy had a slightly increased incidence of hypomagnesaemia than in those without nephropathy ( $94.3 \% \mathrm{v} / \mathrm{s} 0 \%)$. Corsonello et al demonstrated decreased serum magnesium in type $2 \mathrm{DM}$ with nephropathy compared to normal patients. Recent study shows that decreased magnesium concentration is associated with rapid loss of renal function in patients with type 2 DM.

In summary, the present study has demonstrated that hypomagnesaemia is common in type 2 diabetics and magnesium deficiency is conclusively associated with diabetic retinopathy, neuropathy, nephropathy.

Diabetic patients need regular monitoring of serum magnesium concentration. If low level is reported, magnesium supplementation is necessary.

\section{CONCLUSIONS}

1. Prevalence of hypomagnesaemia in type 2 Diabetics is $35 \%$.

2. Prevalence of hypomagnesaemia is higher in patients with microvascular diabetic complications than in those without microvascular complications.

3. Hypomagnesaemia is significantly associated with retinopathy, neuropathy and nephropathy.

4. No significant association was seen with ischaemic heart disease and hypomagnesaemia.

5. No significant association was seen with hypertension and hypomagnesaemia.

6. Prevalence of hypomagnesaemia was high in patients with fasting blood sugar $>131 \mathrm{mg} / \mathrm{dL}$.

7. No significant differences were seen among both sexes.

8. No significant association of hypomagnesaemia was seen with duration of diabetes and HbA1c levels.

\section{Summary}

Present study was conducted to estimate the prevalence of hypomagnesaemia in type 2 diabetics and to study the possible association of hypomagnesaemia with diabetic complications and comorbidities.

The study included 100 type 2 diabetic cases, with no factors significantly altering the serum magnesium levels. Fasting serum magnesium levels were estimated and correlation was done with variable parameters.

The results found that prevalence of low magnesium levels $(<1.7 \mathrm{mg} / \mathrm{dL})$ in type 2 diabetic cases were $35 \%$ and magnesium deficiency was significantly associated with diabetic retinopathy, neuropathy and nephropathy. No significant correlation was identified with other parameters IHD, hypertension, duration of diabetes and treatment modality. Causes of hypomagnesaemia are multifactorial. Available data suggests that low magnesium levels are associated with adverse clinical outcomes. So it is prudent in clinical practice to periodically monitor plasma magnesium concentrations in diabetic patients and have the condition corrected whenever possible.

\section{REFERENCES}

[1] Wild S, Roglic G, Green A, et al. Global prevalence of diabetes: estimates for the year 2000 and projections for 2030. Diabetes Care 2004;27(5):1047-53.

[2] Colledge NR, Walker BR, Ralston SH. Davidson's principles \& practice of medicine. 21 $1^{\text {st }}$ edn. Elsevier Health Sciences UK 2010:795-834.

[3] Ramachandran A, Mary S, Yamuna A, et al. High prevalence of diabetes and cardiovascular risk factors associated with urbanization in India. Diabetes Care 2008;31(5):893-8.

[4] Riduara RL, Stamfer MJ, Willet WC, et al. Magnesium intake and risk of type 2 diabetes in men and women. Diabetes care 2004;27(1):134-40.

[5] Huerta MG, Holmes VF, Romenich JN, et al. Magnesium deficiency is associated with insulin resistance in obese children. Diabetes care 2005;28(5):1175-81.

[6] Yajnik CS, Smith RF, Hockaday TD, et al. Fasting plasma magnesium concentration and glucose disposal in diabetes. BMJ 1984;288(6423):1032-4.

[7] Hatwal A, Gujral AS, Bhatia RP, et al. Association of hypomagnesemia with diabetic retinopathy. Acta Ophthalmol 1989;67(6):714-6.

[8] Sasaki S, Oshima T, Matsuura H, et al. Abnormal magnesium status in patients with cardiovascular diseases. Clin Sci (Lond) 2000;98(2):175-81.

[9] Paolisso G, Passariello N, Pizza G, et al. Dietary magnesium supplements improve B-cell response to glucose and arginine in elderly non-insulin dependent diabetic subjects. Acta endocrinol 1989;121(1):16-20.

[10] Nadler JL, Buchanan T, Natarajan R, et al. Magnesium deficiency produces insulin resistance and increased thromboxane synthesis. Hypertension 1993;21(6 Pt 2):1024-9.

[11] Walti MK, Zimmermann MB, Hurrell RF, et al. Low plasma magnesium in type 2 diabetes. Swiss Med Wkly 2003;133(19-20):289-92.

[12] Schlienger JL, Grunenberger F, Maier EA, et al. Disturbances of plasma trace elements in diabetes. Relation to blood glucose equilibrium. Presse Med 1988;17(21):1076-9.

[13] Alzaid AA, Dinneen SF, Moyer TP, et al. Effects of insulin on plasma magnesium in noninsulindependent diabetes mellitus: evidence for insulin resistance. J Clin Endocrinol Metab 1995;80(4): 1376-81.

[14] Liao F, Folsom AR, Brancati FL. Is low magnesium concentration a risk factor for coronary heart disease? The atherosclerosis risk in communities (ARIC) study. Am Heart J 1998;136(3):480-90.

[15] Grafton G, Bunce CM, Sheppard MC, et al. Effect of $\mathrm{Mg} 2+$ on $\mathrm{Na}(+)$-dependent inositol transport. Role for $\mathrm{Mg} 2+$ in etiology of diabetic complications. Diabetes 1992;41(1):35-9. 